Original Paper http://ajol.info/index.php/ijbcs http://indexmedicus.afro.who.int

\title{
Distribution et diversité de la faune aviaire de la ville de Niamey et de sa principale zone humide, le fleuve Niger
}

\author{
Youssoufa ISSIAKA $^{1 *}$, Soumaila HASSANE HAMANI ${ }^{2}$, Salamatou \\ ABDOURAHAMANE ILLIASSOU ${ }^{3}$ et Aboubacar AWAISS ${ }^{4}$ \\ ${ }^{1}$ Université Dan Dicko Dankoulodo de Maradi, Faculté d'Agronomie et des Sciences de l'Environnement, \\ Département Génie Rural et Eaux et Forêts, BP 465, Maradi, Niger. \\ ${ }^{2}$ Université Dan Dicko Dankoulodo de Maradi, Faculté des Sciences et Techniques, Département de Biologie. \\ BP 465, Maradi, Niger. \\ ${ }^{3}$ Université de Diffa, Faculté des Sciences Agronomiques, Département de production Animale, BP 78, Diffa, \\ Niger. \\ ${ }^{4}$ Université Abdou Moumouni de Niamey, Faculté d'Agronomie, Département Génie Rural et Eaux et Forêts. \\ BP 10960, Niamey, Niger. \\ *Auteur correspondant ; E-mail : youssoufa_maiga@yahoo.fr, Tel: 00227 90815313, Cel: 0022796264035
}

\section{RESUME}

L'urbanisation des villes altère la naturalité des espaces qu'elles occupent. Cette situation impacte surement la diversité et la distribution de la faune aviaire. Ce travail, le premier du genre dans Niamey, a pour objectif d'apprécier la diversité et la distribution de l'avifaune en lien avec les différents types d'habitations de la ville de Niamey et de sa zone humide.

La méthode consiste à dénombrer les oiseaux sur des transects qui passent dans chaque commune et sur le point fixe choisi sur le Fleuve. Les dénombrements se font de 7 h à 9 h30 et de 16 h30 à 18 h30 sur l'ensemble des sites. Cela a permis de recenser 38 espèces pour toute la ville de Niamey avec respectivement 18,22, 18 et 21 espèces sur les transect 1,2, 3 et le fleuve.. Les résultats montrent que les espèces les plus ubiquistes sur les trois transects sont : Streptopelia senegalensis, Lamprotornis purpureus, Bubulcus ibis. Les moins observées sont: Milvus migrans, Merops orientalis, Sylvietta brachyura, Nilaus afer, Uraeginthus bengalus. Sur le fleuve, les plus ubiquistes sont : Bubulcus ibis et Ardea cinerea et les moins observées sont: Ardea alba, Ardeola ralloides, Actophilornis africana, Tringa nebularia et Melanitta nigra.

() 2018 International Formulae Group. All rights reserved

Mots clés : Dénombrement, oiseaux, transect, habitations, zone urbaine, Commune

\section{Distribution and diversity of birdlife in the city of Niamey and its main wetland, the Niger River}




\section{ABSTRACT}

The urbanization of cities alters the naturalness of the spaces they occupy. This situation surely impacts the diversity and distribution of bird fauna. This work, the first of its kind in Niamey, aims to appreciate the diversity and distribution of birdlife in relation to the different types of housing in the city of Niamey and its wetland. The method consists in counting the birds on transects which pass in each commune and on the chosen fixed point on the river. Counts are from $7 \mathrm{am}$ to $9.30 \mathrm{am}$ and from $4.30 \mathrm{pm}$ to $6.30 \mathrm{pm}$ on all sites. This allowed to identify 38 species for the whole city of Niamey with respectively 18,22, 18 and 21 species on transects $1,2,3$ and the river. The results show that the most ubiquitous species on the three transects are : Streptopelia senegalensis, Lamprotornis purpureus, Bubulcus ibis. The least observed are: Milvus migrans, Merops orientalis, Sylvietta brachyura, Nilaus afer, Uraeginthus bengalus. On the river, the most ubiquitous are: Bubulcus ibis and Ardea cinerea and the least observed are: Ardea alba, Ardeola ralloides, Actophilornis africana, Tringa nebularia and Melanitta nigra.

(C) 2018 International Formulae Group. All rights reserved

Keywords: Enumeration, birds, transect, habitations, urban area, Commune.

\section{INTRODUCTION}

Les zones urbaines abritent une grande proportion (environ 50\%) de la population humaine du monde (Lougbegnon et Codjia, 2011) qui augmente rapidement, en particulier dans les pays en voie de développement. Dans ces pays, les paysages urbains hébergent une portion substantielle de diversité biologique globale. Beaucoup de villes croissent rapidement tant en superficie qu'en population (Turner, 2003). Ainsi les préoccupations écologiques en milieux urbains deviennent de plus en plus croissantes aux vues des conséquences que génèrent les activités humaines sur la santé des citadins et leur environnement (Lougbegnon et Codjia, 2011). Les niveaux de perturbations en milieu urbain offrent la possibilité pour diverses catégories d'avifaune aussi bien pour les espèces autochtones fidèles à l'habitat indigène comme pour les espèces opportunistes (ou introduites) de supporter ou d'exploiter ces habitats modifiés (White et al., 2005). La végétation domestique, c'est-à-dire végétation présente dans les parcelles habitées présente des enjeux socio-culturels, économiques et environnementaux (Kouagou Raoul Sambien et al., 2018). Les villes sont donc des milieux intéressants pour certaines espèces d'oiseaux du fait qu'elles leur offrent certaines conditions favorables. En effet, elles procurent à des oiseaux des facilités de nidification (hirondelles par exemple), une quantité appréciable de nourritures, surtout dans les jardins, les dépotoirs (déchets ménagers) et les champs environnant. Les granivores trouvent leurs nourritures partout où l'on manipule des grains (maïs, riz...,). Les insectes ne sont pas absents des grandes villes, ne serait-ce que dans les couches basses de l'atmosphère où chassent les martinets et les hirondelles et dans les champs où des oiseaux insectivores jouent le grand rôle de protéger les cultures contre les insectes ravageurs. Les villes offrent aussi aux oiseaux des microclimats beaucoup plus favorables que les districts avoisinants en raison de leurs vents moins violents et des températures élevées. Elles procurent aux oiseaux une protection contre les prédateurs qui s'adaptent mal à l'urbanisation car ils sont assez farouches et ne sont guère appréciés par l'homme (Ndayikengurukiye, 2005). En milieu urbain apparaissent d'importantes transformations biologiques qui modifient l'écologie des oiseaux. Akobi et al (2018) ont prouvé que la pression anthropique sur le couvert végétal révèle une évolution régressive des formations végétales naturelles au profit des formations anthropiques. Les oiseaux sont des véritables 
indicateurs biologiques qui peuvent nous renseigner sur l'état et le bon fonctionnement des écosystèmes. En effet, les oiseaux constituent un groupe zoologique aisément utilisable en matière de diagnostic écologique capable de déboucher sur des stratégies de conservation favorable à d'autres espèces de la flore et de la faune (Sinsin et Kampman, 2010). En outre, les oiseaux sont un élément visible de la faune urbaine, ce qui signifie que, les résultats des recherches sur l'avifaune en ville, peuvent être restitués aux résidents et aux décideurs pour des prises de décisions. De même, l'effet de l'urbanisation sur cette faune peut être utilisé pour investiguer sur les facteurs qui influencent la distribution, l'abondance et le statut de la conservation de la faune urbaine.

Cette étude a pour objectif global d'apprécier la diversité et la distribution de l'avifaune de la ville de Niamey en plein essor d'urbanisation et de sa principale zone humide qu'est le fleuve Niger.

De manière spécifique il s'agit de:

- Apprécier la dynamique de l'urbanisation de la ville de Niamey ;

- Identifier et dénombrer les oiseaux rencontrés actuellement dans la ville de Niamey (dans les communes I, II et V);

- Comparer la dynamique aviaire des différents types d'habitations.

Les hypothèses suivantes ont été posées pour bien mener cette étude: La ville regorge une importante diversité aviaire dont la distribution suit un gradient d'urbanisation. Cette urbanisation a des effets aussi bien positifs que négatifs sur la distribution et la diversité aviaire.

\section{MATERIEL ET METHODES}

\section{Localisation de la ville de Niamey}

La ville de Niamey est située dans la partie ouest du Niger entre les parallèles $13^{\circ} 33^{\prime}$ nord et $13^{\circ} 24^{\prime}$ sud et les méridiens $2^{\circ}$ et $29^{\circ} 15^{\prime}$ 'Est (Figure 1). Elle est située aux confins du fleuve Niger, à l'extrême-ouest du pays et aujourd'hui en pleine modernisation.

\section{Découpage de la ville}

La Figure 2 ci-dessous montre le découpage administratif de la ville de Niamey en cinq Commune dont quatre sur la rive gauche et une sur la rive droite.

\section{Population}

La population connaitra une croissance rapide et triple en 12 ans passant de 33816 habitants en 1960 à 1011277 en 2012. La chasse, la destruction de l'habitat des oiseaux sont des impacts de cette croissance démographique.

\section{Caractéristiques physiques de la CUN Climat}

Les précipitations sont importantes en août avec des grandes variations dans l'espace et dans le temps. Les pluies se présentent dans la plupart des cas sous forme d'orages. Ces orages peuvent détruire le nid de certains oiseaux. Les cumuls annuels des pluies de 2006 à 2012 sont donnés par la Figure 3.

\section{La végétation}

On rencontre des espèces locales telles que : Acacia nilotica, Bauhinia refescens, Pourparcia birrea, Delonix regia, Adansonia digitata(baobab), Khaya sengalensis (caïcédrat), Acacia seyal, Balanites aegyptiaca, Ceiba pentandra, Prosopis africana, Ziziphus mauritania, Cassia sieberiane, Ficus sp., Hyphaene thebaica(doum) et Parkia biglobosa (Néré). Les espèces exotiques, plantées, sont essentiellement le neem (Azadirachta indica), l'eucalyptus (Eucalyptus camaldulensis), le mélina (Gmelina arborea), Terminalia mentaly, Prosopis juliflora, Cassia siamea, Acacia holocericea. Cette végétation constitue aussi un habitat pour les oiseaux.

\section{Présentation de la ville de Niamey}

La Commune Urbaine de Niamey (CUN) est créée par décret $\mathrm{N}^{\circ} 88$ 393/PCMS/MI du 24 novembre 1988 (Adamou, 2005). Elle est située entre $13^{\circ} 28$ et $13^{\circ} 35$ de latitude Nord et $2^{\circ} 03$ et $2^{\circ} 10$ de longitude Est. D'une superficie de $240 \mathrm{Km}^{2}$, elle est construite sur un plateau surplombant la rive gauche du fleuve Niger et sur une plaine alluviale sur la rive droite, entre 180 et 250 mètres d'altitude. La ville de Niamey est divisée en deux par le fleuve Niger (Figure 
2) : la rive droite et la rive gauche. Elle est subdivisée en cinq communes : les communes I, II, III, et IV se trouvent toutes sur la rive gauche du fleuve tandis que la commune $\mathrm{V}$ se trouve sur la rive droite.

La région de Niamey présente un climat de type sahélo-soudanien caractérisé par de fortes températures, une pluviométrie faible et irrégulière. Le climat de la région est marqué par l'alternance de deux masses d'air, continentales sèches et océaniques humides limitées par une ligne de contact, le front intertropical (FIT) se déplaçant du nord au sud avec la position saisonnière du soleil. L'essentiel des précipitations intervient avec une extrême régularité entre juin et septembre.

Les formations $\mathrm{du}$ Continental qui caractérisent la Région de Niamey, sont surtout argileuses et plus on s'approche du fleuve, plus le socle est proche et la nappe discontinue.

Niamey appartient à la bande sahélienne caractérisée par une alternance de savane arbustive claire et de brousse tigrée. Les abords de la ville, dans la limite de la Communauté Urbaine, présentent des parcs arborés à Faidherbia albida et la majeure partie de la zone est cultivée (mil, sorgho). Les terres, compte tenu des cultures, du pâturage, du déboisement et de la réduction des temps de jachères, ne présentent qu'une végétation naturelle peu dense et caractéristique de sols très dégradés. Harouna et Machiels (1999) signalaient qu'en 1973 déjà une disparition quasi-irréversible de la végétation spontanée autour de Niamey commença. La végétation en ville est essentiellement constituée par la ceinture verte, de petits cordons arborés et des plantations d'arbres fruitiers le long du fleuve et de la vallée du Gounti-Yéna, des plantations dans les concessions ou en bordure de grandes avenues et enfin quelques jardins publics et espaces verts aménagés par la municipalité.

La faune des villes au Niger est trop peu connue. Pour la ville de Niamey, cette étude constitue une première et permettra de mettre à disposition des connaissances sur la faune (aviaire surtout) de la plus grande ville $\mathrm{du}$ pays. La population de Niamey est composée des communautés des huit ethnies du pays avec une prédominance des DjermaSonraï qui sont parmi les premiers habitants de la ville de Niamey avant qu'elle ne devienne capitale du Pays (Tini, 2003).

\section{Matériel technique et organisation des travaux}

Le matériel technique est constitué d'une paire de jumelles pour l'observation des oiseaux, un compteur manuel pour le comptage des grands groupes d'oiseaux, des guides d'identification tels que le Guide des oiseaux de l'Afrique de l'Ouest de Nik Borrow et Ron Demey (2015) et les clefs d'identification des oiseaux (OMPO, 2000). Et enfin un appareil photographique est utilisé pour la prise des photos.

\section{Méthodologie \\ Dénombrement des oiseaux}

Le dénombrement est fait suivant deux méthodes. La première méthode repose sur les transects linéaires. Trois transects d'une longueur moyenne de $3 \mathrm{Km}$ chacun ont été définis dans la commune urbaine de Niamey. Au moins un quartier de chaque Commune est traversé par un de ces transects. Cette méthode consiste à se déplacer le long $\mathrm{du}$ transect et enregistrer le maximum d'individus et d'espèces observés. La seconde méthode est basée sur les points fixes et consiste à dénombrer les oiseaux observés ou entendus à l'intérieur d'un cercle imaginaire de rayon fixe ou limité centré sur l'observateur. Cette méthode a été utilisée au niveau du fleuve. Pour cela nous avons pris trois (3) points dont l'amont (goudel), le centre (pont Kennedy), et l'aval (saga).

Il a été procédé au dénombrement en comptant les individus d'oiseaux et en identifiant les espèces. Pour cela la technique de recensement utilisée par Yaokokoré (2001) et Issiaka (2002 et 2011) a été retenue. Elle consiste à faire le recensement de deux manières: Le recensement le long des transects et les points fixes est effectué le matin de 07 h00 à 09 h30 et le soir de 16 h30 à 18 h30. 
Cinq sessions par transect ont été effectuées dont deux (2) dans le mois de septembre et trois (3) dans le mois d'octobre.

\section{Enquête}

Pour compléter les données de dénombrement, nous avons procédé à des enquêtes auprès des populations locales, hommes et femmes âgés d'au moins 30 ans résidants dans la zone depuis au moins une dizaine d'années. Dix-huit personnes ont été enquêtées dans le premier transect, 18 autres dans le deuxième et 17 dans le troisième. Cette enquête aidera à avoir des informations sur les aspects ci-dessous : (i) Données sur les oiseaux au niveau des habitations traversées par les différents transects. (ii) Importance écologique, économique et culturelle de ces oiseaux selon la conception des populations locales.

\section{Traitement des données}

\section{Calcul des fréquences et abondances}

Les fréquences et indices qui permettent d'apprécier la diversité et les abondances des oiseaux sont calculés à l'aide du Tableur Excel. Ainsi les paramètres cidessous ont été déterminés.

- La richesse spécifique $\mathbf{S}$ : nombre d'espèces dans un milieu

- La fréquence générale d'observation Fi qui est la probabilité d'observer une espèce d'oiseau aux points de dénombrement, à l'occasion d'une visite dans une station : $\mathbf{F i}=$ $\mathrm{Ci} / \mathrm{N}$ avec $\mathrm{Ci}$ (somme de tous les contacts avec l'espèce $\mathrm{i}$ dans les $\mathrm{k}$ points de dénombrement) et $\mathrm{N}$ (somme des relevés effectués dans les $\mathrm{k}$ points de dénombrement que compte une station)

- L'abondance relative générale Ag qui est un indice de dominance des individus de chaque espèce d'Oiseaux dans une station: $\mathbf{A g}=$ $\mathbf{E t} / \mathbf{T}$ avec Et (abondance relative générale) et $\mathrm{T}$ (somme des effectifs moyens des individus de différentes espèces enregistrées dans une station).

- Indice de diversité de Shannon-Weaver: $\mathrm{H}^{\prime}=-\sum \mathrm{pi} \operatorname{lnpi}=-\sum[(\mathrm{ni} / \mathrm{N}) \times \ln (\mathrm{ni} / \mathrm{N})], \mathrm{H}^{\prime} \max =\ln \mathbf{S}$ avec H' (indice de diversité de Shannon); Pi (proportion relative de l'espèce $\mathrm{i}$ dans la communauté) ; ln (logarithme népérien) ; ni (nombre d'individus de l'espèce i) ; $\mathrm{N}$ (nombre total d'individus constituant l'échantillon).

La valeur de l'indice de diversité, communément rencontrée dans les échantillons ou les inventaires d'un groupe zoologique ou botanique étudié, est comprise entre 1 bit et 4,5 bits ou plus dans le cas d'échantillon de grande taille d'une communauté complexe (Toudjani, 2012). Selon le même auteur, l'indice de diversité maximale (ou entropie maximale) Hmax pour des échantillons constitués de 100 à 200 espèces seraient de l'ordre de 8 à 9 .

- L'indice d'équitabilité de Piélou permet d'estimer la dominance d'une espèce. Il varie de 0 à 1 , il tend vers 0 lorsqu'il y a dominance et vers 1 si un maximum d'espèces participe. Il se calcule par la formule suivante : $\mathbf{J}=$ H'/H'max

\section{Caractérisation du peuplement d'oiseaux d'eau}

La caractérisation permet de faire une approche de la structure du peuplement (Issiaka, 2011). Cette caractérisation tient compte de l'abondance des espèces.

Ainsi selon les normes une espèce d'Oiseau est dite :

- Très abondante (TA), si un nombre d'individus supérieurs à 100 peut être vu ou entendus dans l'habitat adéquat chaque jour, Abondante (A), si ce nombre est compris entre 11 et 100 ;

- Commune (C), si ce nombre est compris entre 1 et 10 , Fréquente $(\mathrm{F})$, si l'espèce a été vue souvent mais pas chaque jour, Pas commune (PC), si plusieurs observations sont faites par an, Rare (R), si une observation est faite sur plusieurs années (espèces résidente), Erratique (E), si une observation est faite sur plusieurs années (espèce non résidente). 


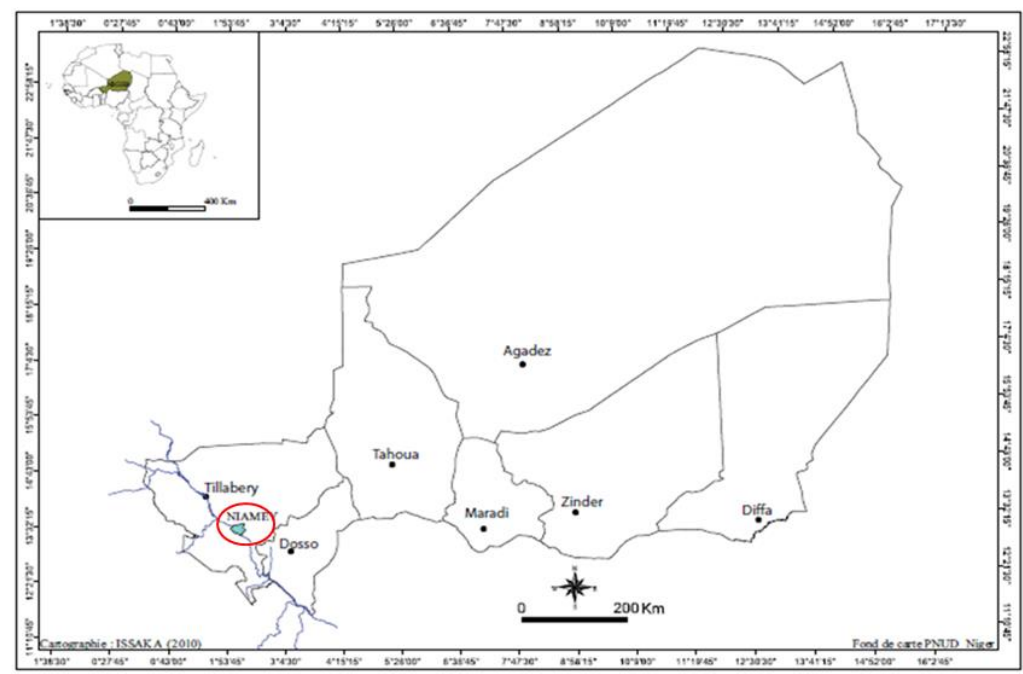

Figure 1 : Carte de localisation de la Commune Urbaine de Niamey (Issaka, 2010).

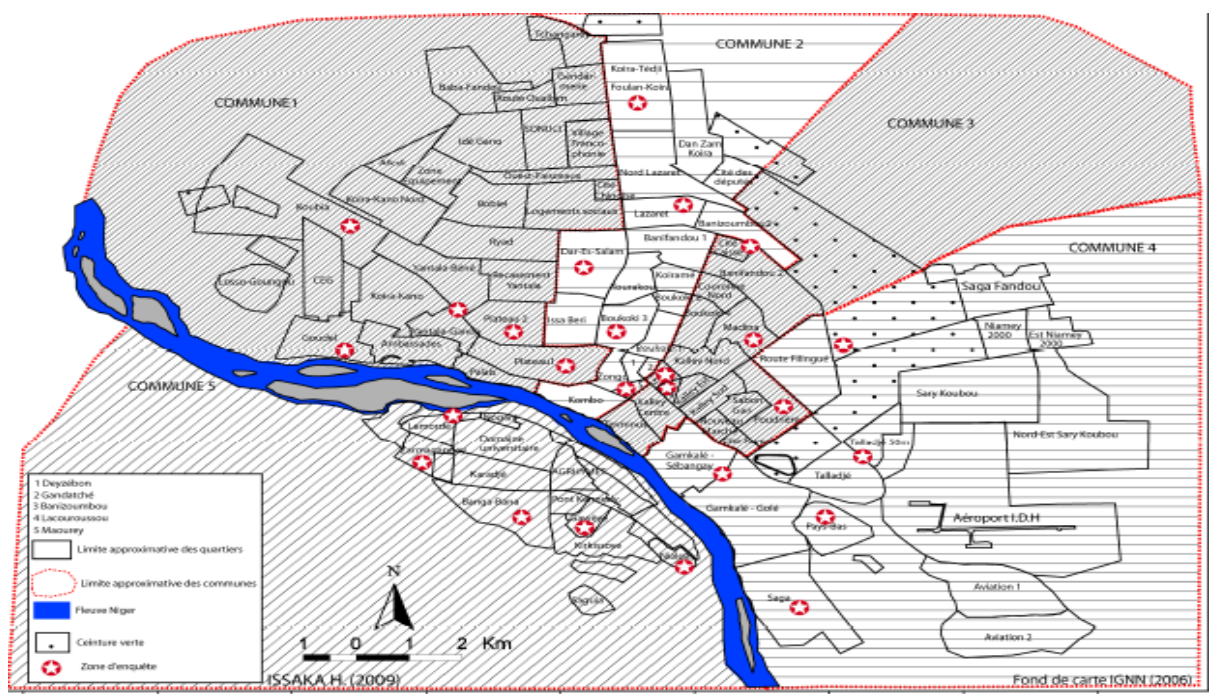

Figure 2 : Subdivision administrative de Niamey (Issaka, 2010).

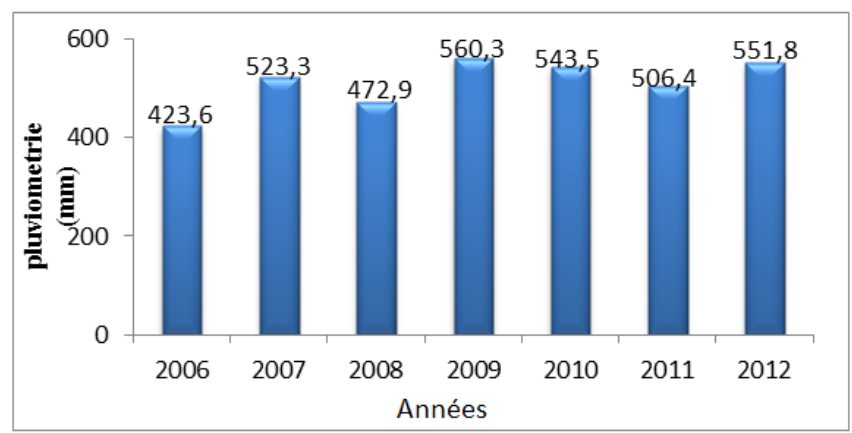

Figure 3 : pluviométrie de la ville de Niamey de 2006 à 2012. Source : DMN, 2012. 


\section{RESULTATS \\ La dynamique de l'urbanisation de la ville de Niamey}

En 1988, la superficie de Niamey était de 4848 ha, à plus de 11000 ha au début des années 2000 et près de $250 \mathrm{~km}^{2}$ actuellement. La superficie agglomérée de la ville de Niamey qui n'était que de 820 hectares en 1960, date d'accession à l'indépendance du Niger, est passée à 2460 hectares en 1977, pour atteindre 5500 hectares en 1984. En 2001, la ville de Niamey s'étale sur un peu plus de 10000 hectares.

En 1988 Niamey comptait 397437 d'habitants, en 2001 il comptait 725030 d'habitants, en 2011 la CUN comptait 707 951 habitants dont 50,64\% d'hommes et $49,36 \%$ de femmes, soit un rapport de masculinité de 102,6 et en 2012 il comptait 1 011277 dont 501459 hommes et 509818 femmes.

\section{Typologie de l'avifaune sur les transects}

Les dénombrement effectués sur les transects ont permis d'enregistrer 18 espèces d'oiseaux sur le transect 1 (rive droite), 22 espèces sur le transect 2 (plateau, yantala ,koirakano) et 18 sur le transect 3 (gountouyena, lazaré). Il faut noter que certaines espèces n'ont été rencontrées que sur un seul de ces transects. On peut citer parmi elles le Salecto à bec blanc (Bubalornis albirostris) sur le transect 1; le Crombec sitelle (Sylvietta brachyura,), le Beau marquet melba, (Pytilia melba), le Cordon bleu à joues rouges (Uraeginthus bengalus), et le Héron cendré (Ardea cinerea) sur le transect 2. Le nombre d'espèces par session ainsi que le nombre total d'espèces par transect est donné par le Tableau 1.

Les espèces recensées sur le transect 1 au cours des cinq sessions se répartissent dans 12 familles. Sur ce transect la famille de Columbidae et celle de Ploceidae sont les plus représentées avec chacune trois (3) espèces et les autres familles sont représentées chacune par une (1) seule espèce. Sur le transect 2, 13 familles sont recensées pour l'ensemble des sessions. Les Columbidae, les Accipitridae et les Estrildidae sont les plus représentées avec chacune trois (3) espèces et les autres ne comptent qu'une seule espèce chacune. Au niveau du transect 3 , les espèces recensées au cours des cinq sessions sont reparties dans 13 familles. La famille des Columbidae est la plus représentée sur ce transect avec trois (3) espèces et les familles les moins représentées ont chacune une seule espèce.

Concernant les effectifs d'oiseaux, le nombre d'individus varie en fonction des sessions sur les transects. Le Tableau 2 donne les résultats de ces dénombrements.

On constate que les effectifs totaux par sessions sont plus importants sur le transect 2 (1141 pour la $1^{\text {ère }}$ session, 812 pour la $2^{\text {ème }}$, 708 pour la $3^{\text {ème }}, 596$ pour la $4^{\text {ème }}$, et 458 pour la $5^{\text {ème }}$ ) que sur les deux autres transects. Les photos 1 et 2 montrent les espèces qui ont plus individus sur le 3 transects linéaires.

\section{Avifaune sur le point fixe (sur le fleuve)}

21 espèces d'oiseaux ont été dénombrées sur ce site. Les espèces les plus fréquemment rencontrées sont Bubulcus ibis, Ardea cinerea, Streptopelia senegalensis, Streptopelia vinacea, Vidua chalybeata, Lagonosticta senegala, et Lamprotornis purpureus. Les détails de ces résultats sont donnés dans les Tableaux 3, 4 et 5 .

\section{Fréquences et indices de diversités Fréquences sur les trois transects}

Le Tableau 6 montre que Streptopelia senegalensis est l'espèce la plus observée avec une fréquence d'observation de 8 sur le premier transect, 6,14 sur le transect 2 et 8,69 sur le transect 3 . Cette espèce est suivie sur le transect 1 de Lamprotornis purpureus avec une fréquence de 3,15 et Bubulcus ibis avec une fréquence de 2,73. Elle est suivie sur le transect 2 Bubulcus ibis avec une fréquence de 3,57 et Lamprotornis purpureus avec une fréquence de 3,39. Sur le transect 3, elle est suivie de Lamprotornis purpureus avec une fréquence de 2,72 Bubulcus ibis avec une fréquence de 2,22. Il faut noter sur le transect1 les espèces les moins fréquentes sont Milvus migrans et 
Merops orientalis avec chacune une fréquence de 0.03 . Sur le transect 2 les espèces le moins fréquentes sont Milvus migrans, Sylvietta brachyura, Nilaus afer et Uraeginthus bengalus avec chacune une fréquence de 0,03 et sur le transect3 l'espèce la moins fréquente est Nilaus afer avec une fréquence de 0,04.

\section{Fréquence sur le fleuve}

Les résultats sur le site du fleuve montrent que Bubulcus ibis est l'espèce la plus observée avec une fréquence de 3,5. Elle est suivie de Ardea cinerea d'une fréquence de 1,1 . Les espèces le moins observées sont Ardea alba, Ardeola ralloides, Actophilornis africana, Tringa nebularia et Melanitta nigra avec chacune une fréquence de 0,1 .

\section{Abondance relative générale (Ag) sur le trois transects}

Les résultats montrent que Bubulcus ibis, Streptopelia senegalensis et et Lamprotornis purpureus avec des $\mathrm{Ag}$ respectivement $\quad(0,15551$ et 0,5964$)$, $(0,31981$ et 0,0805$),(0,12238$ et 0,119$)$ sont les espèces les plus abondantes sur les deux (2) premiers transects. Alors que sur le troisième transect outre Bubulcus ibis et Streptopelia senegalensis , Vidua chalybeata avec Ag égale 0,0932 en terme d'abondance. Les espèces les moins abondantes sont: Malvus migrans avec $\mathrm{Ag}=0,00068$ et Merops orientalis avec $\mathrm{Ag}=0,00068$ sur le transect 1 , Uraeginthus bengalus avec $\mathrm{Ag}=0,0002$ et Nilaus afer avec $\mathrm{Ag}=0,0003$ sur le transect 2 et Accipiter badius avec $\mathrm{Ag}=0,0077$ sur le transect 3.

\section{Abondance relative générale des espèces sur le site du fleuve}

Les résultats du tableau10 montrent que Bubulcus ibis est l'espèce la plus abondante avec une $\mathrm{Ag}$ de 0,7815. Cette espèce est suivie de Ardea alba $\mathrm{Ag}=0,0243$ et Vidua chalybeata $A g=0,0229$ mais à des abondances beaucoup moins faibles que Bubulcus ibis. Tandis que Melanitta nigra $\mathrm{Ag}$ 0,0013, Alopochena egyptiaca Ag 0,004 et Streptopelia vinacea Ag 0,0047, constituent les espèces les moins rencontrées sur le fleuve.

\section{Diversités sur les trois transects}

Le Tableau 7 montre que, pour les espèces d'Oiseaux, l'indice de diversité varie d'une session à une autre et d'un transect à un autre.

L'indice d'équitabilité de Piélou varie d'une session à une autre et d'un site à un autre et il est plus grand sur l'ensemble des sessions pour les transects 1 et 3 (Tableau 8).

\section{Diversités sur le site du fleuve}

La Figure 4 montre que l'indice de Shannon varie d'une session à une autre et il est plus élevé au niveau de la première et plus faible pour la deuxième session.

La Figure 5 montre que l'indice d'équitabilité de Piélou varie d'une session à une autre. Comme l'indice de Shannon, il est aussi élevé au niveau de la première session et plus faible niveau de la deuxième session.

\section{Caractérisation du peuplement d'oiseaux sur les transects}

Le peuplement d'oiseaux sur les sites d'étude se caractérise par un faible nombre d'espèces abondantes (Tableau 9).

Sur le point fixe du fleuve, Bubulcus ibis est la seule espèce abondante et Egretta alba est commune. Toutes les autres espèces de ce site ont un statut fréquent.

\section{Résultats des enquêtes}

$\mathrm{Au}$ niveau du transect 1,18 personnes ont été enquêtées (hommes et femmes). Selon 14 d'entre elles $(77,77 \%)$, les nombres d'espèces d'oiseaux diminuent dans la ville de Niamey depuis quelques années. Quinze personnes (soit 83,33\%) trouvent que le nombre d'individus d'oiseaux augmente dans la ville de Niamey, et cette augmentation est due selon la plupart de ces gens à la disponibilité de la nourriture et la diminution de la prédation.

Au niveau du transect 2, 18 personnes ont également été enquêtées ; parmi elles 15 admettent une diminution du nombre d'espèces d'oiseaux soit $83,33 \%$ et selon ce même nombre d'enquêtés, il y a eu une 
augmentation du nombre d'individus d'oiseaux.

$\mathrm{Au}$ niveau du troisième transect, 17 personnes ont été enquêtées et selon 14 personnes soit $82,35 \%$ il y'a eu une diminution du nombre d'espèces d'oiseaux de la ville de Niamey. Aussi, et $88,23 \%$ soit 15

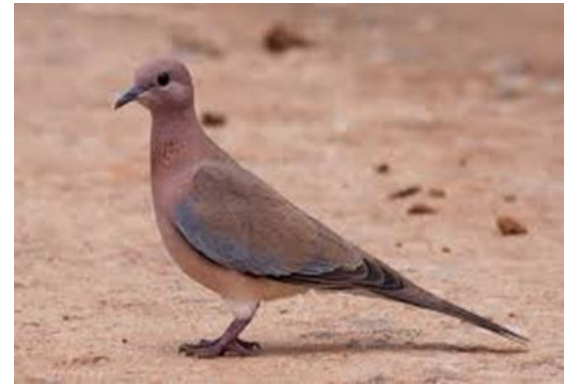

Photo 1 : Tourterelle maillée Streptopelia senegalensis. (Photo 1 :Image Oiseaux.net)

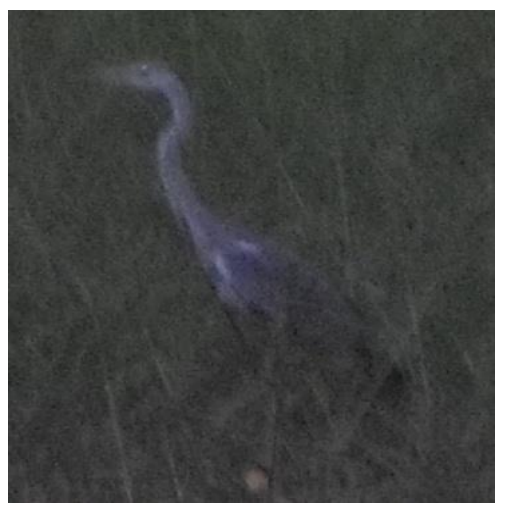

Photo 3 : Héron cendré (Ardea cinerea) personnes constatent une augmentation du nombre d'individus d'oiseaux.

Sur tous ces transects, selon les personnes enquêtées cette diminution du nombre d'espèces d'oiseaux est due soit à la destruction de leurs habitats, soit à la chasse, soit au changement climatique, soit à la démographie.

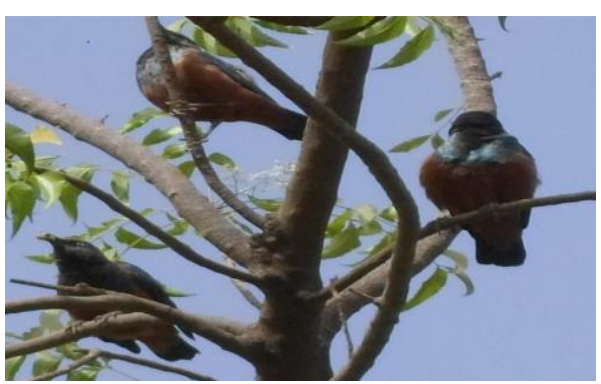

Photo 2 : Choucador pourpré (Lamprotornis purpureus)

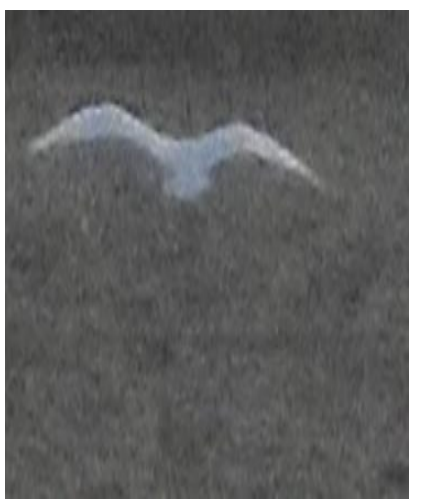

Photo 4 : Héron garde bœufs (Bubulcus ibis) 
Tableau 1 : Nombre d'Espèces d'oiseaux observés par session et par transect.

\begin{tabular}{cccc}
\hline Transect & Sessions & Nombre d'espèces par session & Total des espèces par sites \\
\hline & $1^{\text {ère }}$ & 12 & \\
\hline 1 & $2^{\text {ème }}$ & 13 & 18 \\
\hline & $3^{\text {ème }}$ & 12 & \\
\hline & $4^{\text {ème }}$ & 12 & \\
\hline & $5^{\text {ème }}$ & 14 & \\
\hline & $1^{\text {ère }}$ & 19 & \\
\hline & $2^{\text {ème }}$ & 17 & \\
\hline & $3^{\text {ème }}$ & 17 & \\
\hline & $4^{\text {eme }}$ & 15 & \\
\hline & $5^{\text {eme }}$ & 17 & \\
\hline & $1^{\text {ère }}$ & 15 & \\
\hline & $2^{\text {ème }}$ & 16 & \\
\hline & $3^{\text {ème }}$ & 16 & \\
\hline & $4^{\text {ème }}$ & 16 & \\
\hline & $5^{\text {ème }}$ & 16 & \\
\hline
\end{tabular}

Tableau 2 : Effectifs des espèces d'Oiseaux par session et par transect. (T1, T2, T3 désignent respectivement les transects 1,2 , et 3 ).

\begin{tabular}{lllllllllllllllll}
\hline Sessions & \multicolumn{1}{l}{ 1ere session } & \multicolumn{3}{l}{ 2eme session } & \multicolumn{3}{c}{ 3eme session } & \multicolumn{3}{c}{ 4eme session } & \multicolumn{3}{c}{ 5eme session } \\
\hline Especes & $\mathrm{T} 1$ & $\mathrm{~T} 2$ & $\mathrm{~T} 3$ & $\mathrm{~T} 1$ & $\mathrm{~T} 2$ & $\mathrm{~T} 3$ & $\mathrm{~T} 1$ & $\mathrm{~T} 2$ & $\mathrm{~T} 3$ & $\mathrm{~T} 1$ & $\mathrm{~T} 2$ & $\mathrm{~T} 3$ & $\mathrm{~T} 1$ & $\mathrm{~T} 2$ & $\mathrm{~T} 3$ \\
\hline Bubulcus ibis & 35 & 445 & 45 & 20 & 467 & 28 & 25 & 499 & 30 & 38 & 403 & 27 & 12 & 275 & 32 \\
\hline Ardea cinerea & 0 & 61 & 0 & 0 & 42 & 0 & 0 & 25 & 0 & 0 & 17 & 0 & 0 & 25 & 0 \\
\hline Accipiter badius & 2 & 1 & 2 & 3 & 3 & 1 & 3 & 2 & 1 & 1 & 4 & 2 & 4 & 3 & 3 \\
\hline Necrosyrtes monachus & 0 & 5 & 0 & 0 & 3 & 4 & 0 & 12 & 3 & 0 & 2 & 4 & 0 & 2 & 4 \\
\hline Malvus migrans & 0 & 2 & 0 & 0 & 2 & 0 & 0 & 1 & 0 & 1 & 0 & 0 & 0 & 1 & 0 \\
\hline Streptopella senegalensis & 92 & 124 & 40 & 69 & 49 & 54 & 43 & 40 & 39 & 62 & 60 & 35 & 55 & 41 & 32 \\
\hline Columba guinea & 3 & 5 & 3 & 6 & 9 & 5 & 6 & 5 & 4 & 2 & 6 & 4 & 6 & 5 & 8 \\
\hline Streptopella vinacea & 0 & 7 & 6 & 3 & 6 & 5 & 0 & 6 & 2 & 5 & 5 & 7 & 4 & 5 & 5 \\
\hline Cypsiurus parvus & 11 & 22 & 11 & 19 & 18 & 10 & 0 & 10 & 15 & 5 & 18 & 7 & 5 & 22 & 12 \\
\hline Coracia abyssinicus & 0 & 2 & 3 & 3 & 4 & 2 & 4 & 2 & 3 & 2 & 3 & 4 & 2 & 4 & 3 \\
\hline Merops orientalis & 0 & 0 & 2 & 0 & 0 & 2 & 0 & 0 & 5 & 0 & 0 & 2 & 1 & 0 & 3 \\
\hline Tockus nasutus & 2 & 7 & 9 & 0 & 3 & 9 & 4 & 2 & 10 & 7 & 2 & 11 & 4 & 4 & 7 \\
\hline Nilaus afer & 0 & 0 & 10 & 0 & 0 & 3 & 0 & 0 & 5 & 0 & 0 & 10 & 0 & 2 & 10 \\
\hline Corvus albus & 0 & 12 & 5 & 0 & 11 & 4 & 2 & 8 & 4 & 6 & 3 & 5 & 0 & 0 & 4 \\
\hline Sylvieta brachyura & 0 & 5 & 0 & 0 & 0 & 0 & 0 & 0 & 0 & 0 & 0 & 0 & 0 & 0 & 0 \\
\hline Lamprotornis purpureus & 21 & 284 & 25 & 30 & 92 & 15 & 30 & 48 & 15 & 20 & 20 & 14 & 19 & 30 & 13
\end{tabular}




\begin{tabular}{llllllllllllllll} 
Lamprotornis caudatus & 6 & 5 & 6 & 15 & 6 & 5 & 6 & 2 & 4 & 6 & 4 & 6 & 12 & 5 & 9 \\
\hline Placeus vitellinus & 8 & 7 & 11 & 5 & 9 & 5 & 2 & 4 & 10 & 4 & 3 & 18 & 0 & 4 & 17 \\
\hline Placeus luteolus & 27 & 29 & 19 & 10 & 19 & 10 & 11 & 8 & 18 & 15 & 15 & 24 & 3 & 5 & 20 \\
\hline Bubalornis albirostris & 12 & 0 & 0 & 0 & 0 & 0 & 6 & 0 & 0 & 6 & 0 & 0 & 0 & 0 & 0 \\
\hline Lagonosticta senegala & 4 & 46 & 20 & 15 & 37 & 10 & 9 & 19 & 25 & 4 & 13 & 20 & 6 & 8 & 22 \\
\hline Pytilia melba & 0 & 11 & 0 & 0 & 0 & 0 & 0 & 0 & 0 & 0 & 0 & 0 & 0 & 0 & 0 \\
\hline Uraeginthus bengalus & 0 & 0 & 0 & 0 & 0 & 0 & 0 & 0 & 0 & 0 & 0 & 0 & 0 & 1 & 0 \\
\hline Vidua chalybeata & 30 & 61 & 25 & 22 & 32 & 12 & 12 & 15 & 20 & 20 & 18 & 25 & 5 & 16 & 19 \\
\hline Total & $\mathbf{2 5 3}$ & $\mathbf{1 1 4 1}$ & $\mathbf{2 4 2}$ & $\mathbf{2 2 0}$ & $\mathbf{8 1 2}$ & $\mathbf{1 8 4}$ & $\mathbf{1 6 3}$ & $\mathbf{7 0 8}$ & $\mathbf{2 1 3}$ & $\mathbf{2 0 4}$ & $\mathbf{5 9 6}$ & $\mathbf{2 2 5}$ & $\mathbf{1 3 8}$ & $\mathbf{4 5 8}$ & $\mathbf{2 2 3}$ \\
\hline
\end{tabular}

Tableau 3: Nombre d'espèces d'Oiseaux par session sur le site du fleuve.

\begin{tabular}{cccc}
\hline sites & sessions & nombre d'espèce par session & total \\
\hline \multirow{3}{*}{ Fleuve } & $1^{\text {ère }}$ & 16 & \\
\cline { 2 - 3 } & $2^{\text {ème }}$ & 14 & 21 \\
\cline { 2 - 3 } & $3^{\text {ème }}$ & 17 & \\
\cline { 2 - 3 } & $4^{\text {ème }}$ & 15 & \\
\hline & $5^{\text {ème }}$ & 15 \\
\hline
\end{tabular}

Tableau 4 : Nombre d'espèces par famille sur le site du fleuve.

\begin{tabular}{|c|c|c|c|c|}
\hline Site & nombre de Famille & Famille & Nombre espèces & Especes \\
\hline \multirow{16}{*}{ Fleuve } & \multirow{16}{*}{9} & \multirow{8}{*}{ Ardeidae } & \multirow{8}{*}{8} & Bubulcus ibis \\
\hline & & & & Egretta garzetta \\
\hline & & & & Egretta alba \\
\hline & & & & Egretta intermadia \\
\hline & & & & Ardeola ralloides \\
\hline & & & & Ardea cinerea \\
\hline & & & & Ardea melanocephala \\
\hline & & & & Ardea purpurea \\
\hline & & \multirow{3}{*}{ Anatidae } & \multirow{3}{*}{3} & Melanitta nigra \\
\hline & & & & Dendrocygna viduata \\
\hline & & & & Alopochen aegyptiaca \\
\hline & & \multirow{2}{*}{ Chacadriidae } & \multirow{2}{*}{2} & Vanelus spinosus \\
\hline & & & & Vanelus tectus \\
\hline & & \multirow{2}{*}{ Scolopacidae } & \multirow{2}{*}{2} & Tringa ochropus \\
\hline & & & & Tringa nebularia \\
\hline & & Columbidae & 2 & Streptopelia senegalensis \\
\hline
\end{tabular}




\begin{tabular}{lll} 
& & Streptopelia vinacea \\
\hline Jacanidae & 1 & Actophilornis africana \\
\hline Viduidae & 1 & Vidua chalybeata \\
\hline Estrildidae & 1 & Lagonosticta senegala \\
\hline Sturnidae & 1 & Lamprotornis purpureus \\
\hline
\end{tabular}

Tableau 5 : Effectifs des individus d'Oiseaux par session sur le site du fleuve.

\begin{tabular}{llllll}
\hline Sessions & $\begin{array}{l}\text { 1ère } \\
\text { session }\end{array}$ & $\begin{array}{l}\mathbf{2}^{\text {ème }} \\
\text { session }\end{array}$ & $\begin{array}{l}\mathbf{3}^{\text {ème }} \\
\text { session }\end{array}$ & $\begin{array}{l}\mathbf{4}^{\text {ème }} \\
\text { session }\end{array}$ & $\begin{array}{l}\mathbf{5}^{\text {ème }} \\
\text { session }\end{array}$ \\
\hline Bubulcus ibis & 51 & 108 & 34 & 75 & 98 \\
\hline Egratta garzetta & 8 & 0 & 0 & 4 & 8 \\
\hline Egratta alba & 5 & 6 & 2 & 7 & 5 \\
\hline Egratta intermedia & 3 & 5 & 2 & 0 & 6 \\
\hline Ardeola ralloides & 2 & 4 & 0 & 4 & 3 \\
\hline Actophilornis africana & 5 & 0 & 0 & 0 & 6 \\
\hline Vanellus tectus & 3 & 0 & 4 & 0 & 0 \\
\hline Vanellus spinosus & 4 & 0 & 5 & 3 & 2 \\
\hline Tringa ochropus & 6 & 0 & 5 & 0 & 0 \\
\hline Tringa nebularia & 3 & 0 & 3 & 0 & 0 \\
\hline Ardea cinerea & 4 & 3 & 0 & 5 & 0 \\
\hline Ardea melanocephala & 2 & 0 & 4 & 0 & 0 \\
\hline Ardea purpurea & 3 & 4 & 0 & 5 & 4 \\
\hline Melanitta nigra & 2 & 0 & 0 & 0 & 0 \\
\hline Dendrocygna viduala & 0 & 3 & 0 & 0 & 4 \\
\hline Alopochena egyptiaca & 0 & 0 & 2 & 4 & 0 \\
\hline Streptopelia senegalensis & 6 & 0 & 0 & 4 & 0 \\
\hline Streptopelia vinacea & 2 & 2 & 0 & 0 & 3 \\
\hline Vidua chalybeata & 7 & 5 & 5 & 0 & 7 \\
\hline Lagonosticta senegala & 6 & 0 & 7 & 0 & 0 \\
\hline Lamprotornis purpureus & 10 & 0 & 13 & 0 & 0 \\
\hline Total & $\mathbf{1 3 2}$ & $\mathbf{1 4 0}$ & $\mathbf{8 6}$ & $\mathbf{1 1 1}$ & $\mathbf{1 4 6}$ \\
\hline & & & & 0 & 0 \\
\hline
\end{tabular}


Tableau 6: fréquences générales d'observations des espèces d'Oiseaux par sessions et par transects.

\begin{tabular}{llll}
\hline $\begin{array}{l}\text { Fréquences par transect } \\
\text { Espèces }\end{array}$ & Fi : transect 1 & Fi : transect 2 & Fi : transect 3 \\
\hline Bubulcus ibis & 2.73 & 3.57 & 2.22 \\
\hline Streptopelia senegalensis & 8 & 6.14 & 8.69 \\
\hline Streptopelia vinacea & 0.46 & 0.75 & 0.72 \\
\hline Lamprotornis purpureus & 3.15 & 3.39 & 2.72 \\
\hline Lamprotornis caudatus & 0.38 & 0.78 & 0.86 \\
\hline Columba guinea & 0.69 & 0.82 & 0.77 \\
\hline Coracia abyssinicus & 0.46 & 0.39 & 0.59 \\
\hline Accipiter badius & 0.69 & 0.5 & 0.27 \\
\hline Tockus nasutus & 0.46 & 0.07 & 0.90 \\
\hline Malvus migrans & 0.03 & 0.03 & 0 \\
\hline Merops orientalis & 0.03 & 0 & 0.40 \\
\hline Cypsiurus parvus & 0.23 & 0.57 & 0.36 \\
\hline Corvus albus & 0.11 & 0.14 & 0.27 \\
\hline Lagonosticta senegala & 0.88 & 0.85 & 0.54 \\
\hline Vidua chalybeata & 1.15 & 0.75 & 0.68 \\
\hline Bubalornis albirostris & 0.57 & 0 & 0 \\
\hline Placeus luteolus & 0.57 & 0.64 & 0.63 \\
\hline Placeus vitellinus & 0.30 & 0.25 & 0.22 \\
\hline Sylvietta brachyura & 0 & 0.03 & 0 \\
\hline Pytilia melba & 0 & 0.14 & 0 \\
\hline Nilaus afer & 0 & 0.03 & 0.04 \\
\hline Uraeginthus bengalus & 0 & 0.03 & 0 \\
\hline Ardea cinerea & 0 & 0.28 & 0.18 \\
\hline Necrosyrtes monachus & 0 & 0.14 & \\
\hline & & & 0 \\
\hline
\end{tabular}

Tableau 7 : L'indice de Shannon-Weaver par sessions et par transects.

\begin{tabular}{lcc}
\hline Sessions & Indice de Shannon & transects \\
\cline { 1 - 2 } $1^{\text {ère }}$ & 2,013659774 & \\
\cline { 1 - 2 } $2^{\text {ème }}$ & 2,161792072 & 1 \\
\hline $3^{\text {ème }}$ & 2,212642091 & \\
\hline $4^{\text {ème }}$ & 2,227967964 & 2 \\
\hline $5^{\text {ème }}$ & 2,081233769 & \\
\hline $1^{\text {ème }}$ & 1,876657309 & \\
\hline $3^{\text {ème }}$ & 1,661634378 & \\
\hline $4^{\text {ème }}$ & 1,299020147 & \\
\hline $5^{\text {ème }}$ & 1,370311038 & \\
\hline $2^{\text {ème }}$ & 1,621915198 & \\
& 2,471892679 & \\
& 2,403269397 & \\
& 2638 &
\end{tabular}




\begin{tabular}{ll}
$3^{\text {ème }}$ & 2,519371293 \\
\hline $4^{\text {ème }}$ & 2,598725232 \\
\hline $5^{\text {eme }}$ & 2,631029634 \\
\hline
\end{tabular}

Tableau 8 : l'indice d'équitabilité de Piélou par sessions et par transects.

\begin{tabular}{|c|c|c|}
\hline Transect & Sessions & équitabilité de piélou \\
\hline \multirow{5}{*}{1} & 1ère & 0,785068 \\
\hline & 2ème & 0,8428206 \\
\hline & 3ème & 0,8384214 \\
\hline & 4ème & 0,7863749 \\
\hline & 5ème & 0,7685359 \\
\hline \multirow{5}{*}{2} & 1ère & 0,6264436 \\
\hline & 2ème & 0,5546672 \\
\hline & 3ème & 0,4494301 \\
\hline & 4ème & 0,4836597 \\
\hline & 5ème & 0,5508401 \\
\hline \multirow{5}{*}{3} & 1ère & 0,8724697 \\
\hline & 2ème & 0,8314741 \\
\hline & 3ème & 0,8716426 \\
\hline & 4ème & 0,8990972 \\
\hline & 5ème & 0,9102738 \\
\hline
\end{tabular}

Tableau 9 : Statuts des espèces d'oiseaux rencontrées sur les trois transects.

\begin{tabular}{llll}
\hline \multicolumn{1}{c}{ Statut } & Transect 1 & Transect 2 & Transect 3 \\
\hline Bubulcus ibis & $\mathrm{A}$ & $\mathrm{TA}$ & $\mathrm{A}$ \\
\hline Streptopelia senegalensis & $\mathrm{A}$ & $\mathrm{A}$ & $\mathrm{A}$ \\
\hline Lamprotornis purpureus & $\mathrm{A}$ & $\mathrm{A}$ & $\mathrm{A}$ \\
\hline Lamprotornis caudatus & $\mathrm{C}$ & $\mathrm{C}$ & $\mathrm{C}$ \\
\hline Columba guinea & $\mathrm{C}$ & $\mathrm{C}$ & $\mathrm{C}$ \\
\hline Vidua chalybeata & $\mathrm{A}$ & $\mathrm{A}$ & $\mathrm{A}$ \\
\hline Streptopelia vinacea & $\mathrm{F}$ & $\mathrm{C}$ & $\mathrm{C}$ \\
\hline Placeus luteolus & $\mathrm{A}$ & $\mathrm{C}$ & $\mathrm{A}$ \\
\hline Placeus vitellinus & $\mathrm{C}$ & $\mathrm{C}$ & $\mathrm{A}$ \\
\hline Accipiter badius & $\mathrm{C}$ & $\mathrm{C}$ & $\mathrm{C}$ \\
\hline Coracia abyssinicus & $\mathrm{F}$ & $\mathrm{C}$ & $\mathrm{C}$ \\
\hline Cypsiurus parvus & $\mathrm{F}$ & $\mathrm{A}$ & $\mathrm{C}$ \\
\hline Lagonosticta senegala & $\mathrm{C}$ & $\mathrm{A}$ & $\mathrm{A}$ \\
\hline Necrosyrtes monachus & $\mathrm{R}$ & $\mathrm{C}$ & $\mathrm{C}$
\end{tabular}




\begin{tabular}{llll} 
Corvus albus & $\mathrm{F}$ & $\mathrm{F}$ & $\mathrm{C}$ \\
\hline Tockus nasutus & $\mathrm{F}$ & $\mathrm{C}$ & $\mathrm{C}$ \\
\hline Ardea cinerea & $\mathrm{R}$ & $\mathrm{A}$ & $\mathrm{R}$ \\
\hline Sylvietta brachyura & $\mathrm{R}$ & $\mathrm{F}$ & $\mathrm{R}$ \\
\hline Pytilia melba & $\mathrm{R}$ & $\mathrm{F}$ & $\mathrm{R}$ \\
\hline Nilaus afer & $\mathrm{R}$ & $\mathrm{F}$ & $\mathrm{C}$ \\
\hline Uraeginthus bengalus & $\mathrm{R}$ & $\mathrm{F}$ & $\mathrm{R}$ \\
\hline Malvus migrans & $\mathrm{F}$ & $\mathrm{F}$ & $\mathrm{R}$ \\
\hline Merops orientalis & $\mathrm{F}$ & $\mathrm{R}$ & $\mathrm{C}$ \\
\hline Bubalornis albirostris & $\mathrm{F}$ & $\mathrm{R}$ & $\mathrm{R}$ \\
\hline
\end{tabular}

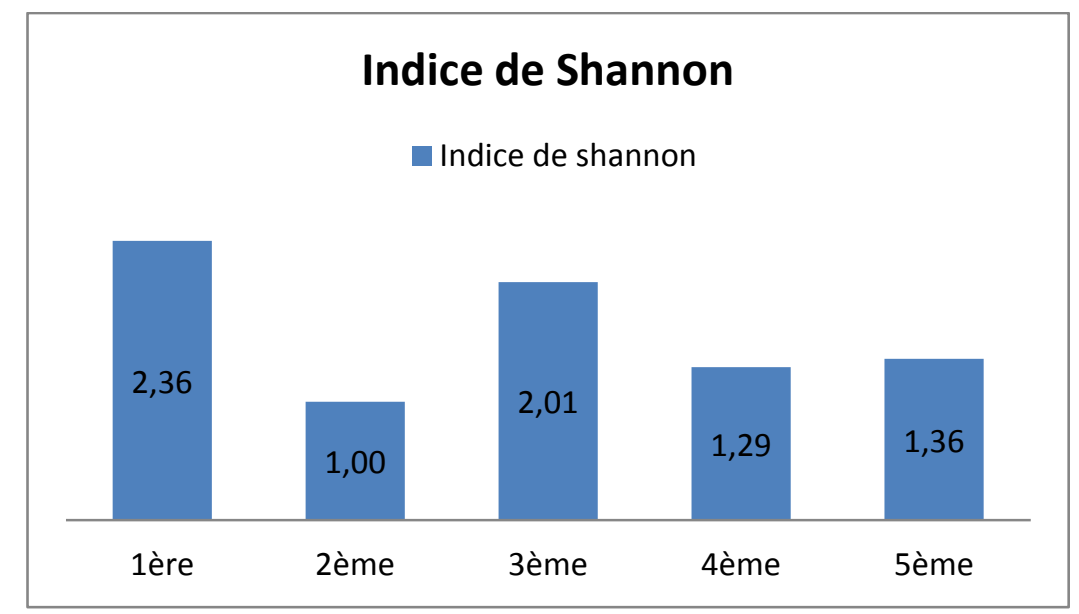

Figure 4: Diversité de Shannon par session.

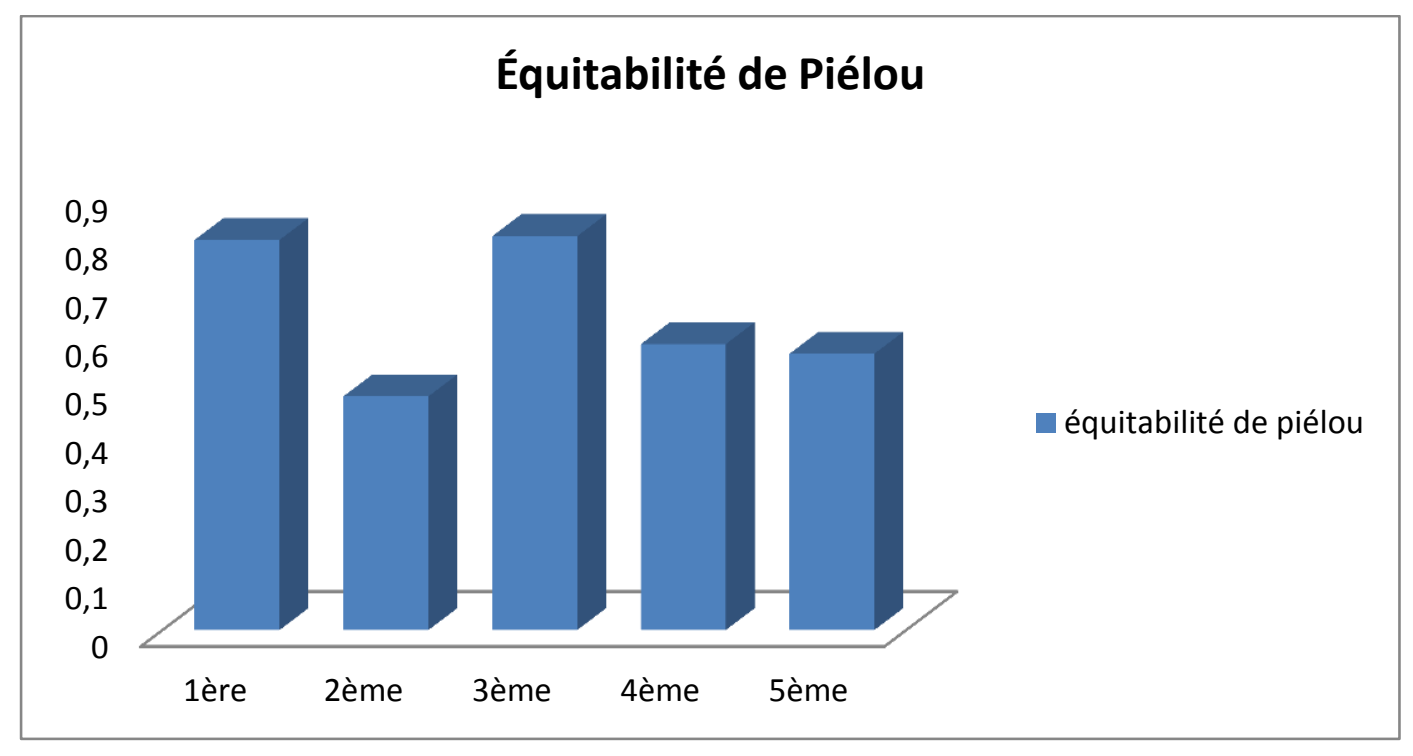

Figure 5 : Indices d'équitabilité de Pielou par session. 


\section{DISCUSSION}

La faune aviaire présente une diversité semblable sur les transect 1 et 3 avec 18 espèces pour chacun. Ces deux transects traversent tous les deux des habitats peu boisés. La plus grande diversité d'oiseau est rencontrée sur les transects boisés comme le 1 où l'on note régulièrement des grands arbres et beaucoup des grands bâtiments qui constituent surement des habitats essentiels pour les oiseaux urbains. 14 des 18 espèces dénombrées sur le transect 1, l'ont été en octobre. Cela est probablement dû au fait que cette 5eme session est effectuée pendant une période de raréfaction des précipitations et coïncide aussi avec le début de retour des oiseaux migrateurs sur le territoire nigérien (Issiaka, 2011). Konan et Yaokokore-Beibro (2015) ont également observé que la grande saison sèche en Côte d'Ivoire correspond à la raréfaction des précipitations et à la période de présence des espèces migratrices du paléarctique. Vingt-deux (22) espèces ont été enregistrées sur le transect 2 (plateau-yantalakoirakano) avec plus d'espèces (19 espèces) pendant la $1^{\text {ère }}$ session effectuée en septembre. Cela est probablement dû au fait que ce transect traverse des habitats très boisés et que cette période coïncide avec la fin de la saison pluvieuse donc un moment favorable aux activités des oiseaux du faite de l'abondance des aliments. Et sur le transect 3 (gountouyena-lazaré) 18 espèces ont été enregistrées avec plus d'espèces (16 espèces) pendant la $4^{\text {ème }}$ session en octobre. Comme pour le transect 1 , cela est probablement dû au fait que cette session est effectuée pendant une période non pluvieuse qui coïncide avec le retour des oiseaux migrateurs. La présence de la zone humide de gountou yena peut aussi expliquer cette diversité aviaire pendant cette période. Pour le point fixe (fleuve), les sessions effectuées ont permis de dénombrer 21 espèces d'oiseaux avec 17 espèces lors de la session du mois d'octobre qui correspond au début de la montée des eaux du fleuve. Toutes les espèces identifiées sur les quatre sites de dénombrement ont été signalées par Denis en 2009.

Par contre certaines espèces identifiées dans la ville de Niamey n'ont pas été observées par Issiaka (2011) dans les zones humides du Parc National du W du Niger qui a recensé 57 espèces et Toudjani (2012) qui a recensé 34 espèces sur le Lac de Madarounfa et 29 espèces sur la Mare de Kourfin koura. Cependant, la plus part des espèces recensées sur le transect 4 (fleuve) ont été observées par ces deux auteurs. Cela montre qu'il y a une similitude des espèces qui fréquentent les zones humides au Niger.

Hors du Niger, Lougbegnon et Codjia (2011) ont réalisé des travaux similaires à Cotonou (Benin) et l'ensemble des espèces recensées sur nos quatre habitats figure sur leur liste. Ceci montre une ressemblance de la diversité des espèces d'oiseaux qui occupent les villes en Afrique de l'ouest.

Les valeurs enregistrées pour l'indice de Shannon-Weaver pour les quatre sites montrent qu'il y a participation de plusieurs espèces dans l'occupation de ces sites. Ce résultat est presque similaire à celui de Toudjani (2012) avec entre 1,48 à 2,09 à la mare Kourfin koura et 1,91 à 2,25 au Lac Madarounfa bien que ses résultats concernent uniquement les oiseaux d'eau.

Les indices d'équitabilité de Piélou qui varient de 0,76 à 0,84 pour le transect 1 , de 0,44 à 0,62 pour le transect 2 , de 0,83 à 0,91 pour le transect 3 et de 0,48 à 0,81 pour le transect 4. Cela explique l'absence de dominance d'une espèce sur les autres mais une contribution de chacune d'elle avec une variation des effectifs.

En effet, les résultats de cette étude montrent aussi que Bubulcus ibis, Streptopelia senegalensis, Lamprotornis purpureus et Vidua chalybeata constituent les espèces dominantes et par conséquent les plus observées. Mais seule Bubulcus ibis constitue l'espèce qui est très abondante (TA), comme le montre ses effectifs enregistrés sur le transect 2. De même, sur le fleuve les résultats montrent que seule Bubulcus ibis répond au 
statut d'espèce abondante (A). Cela trouve son explique dans le fait que cette espèce tolère plus ou moins la présence humaine et peut même coloniser des milieux très anthropisés. Il est un compagnon de l'Homme (Berger) nichant parfois dans les arbres en pleine ville et village. Les autres espèces sont rares et peu abondantes sur le fleuve, situation qui s'explique par un début de pollution de certaines zones du fleuve comme l'ont prouvé Alhou et al. (2009). En effet, ces auteurs ont montré que le changement de la qualité des eaux est également traduit en aval de certains points de rejet par la baisse du taux d'oxygène dissous et l'augmentation du $\mathrm{pH}$. Il s'en suit ainsi, un changement dans la composition spécifique de la communauté des macroinvertébrés dont se nourrissent certaines espèces d'oiseaux le long du fleuve.

\section{Conclusion}

Cette étude effectuée au cours de cinq (5) sessions de terrain de septembre à octobre sur quatre sites a permis de connaître l'état de lieux de la distribution et de la diversité aviaire de la ville de Niamey. 38 espèces sont recensées pour toute la ville, 24 sur les transects et 21 sur les points fixes. Le résultat de nos enquêtes sur les différents transects montre que malgré cette richesse aviaire, un certain nombre des menaces ont été constatés: la chasse, la destruction de l'habitat et le changement climatique.

Cette étude est l'une des premières réalisées dans la ville de Niamey sur sa faune aviaire. Ces résultats obtenus peuvent servir de base scientifique pour orienter l'aménagement urbain. Il serait opportun de pousser les recherches sur la dynamique des populations. Un but à long terme des écologistes urbains, devrait être la conservation de la biodiversité et prioritairement des habitats naturels en milieu urbain pour maintenir et renforcer, dans ce monde à forte urbanisation, le contact entre les humains et la nature. Il est nécessaire d'effectuer des études similaires dans toutes les grandes villes du pays pour bien permettre une bonne connaissance de l'avifaune. Pour une bonne gestion de la biodiversité urbaine, cette avifaune a besoin d'une protection efficace et durable.

\section{CONFLIT D'INTERETS}

Les auteurs déclarent qu'il n'y a aucun conflit d'intérêts.

\section{CONTRIBUTIONS DES AUTEURS}

YI a proposé le protocole de recherche et réalisé le premier draft manuscrit. AA a supervisé le travail. SHH, SAI ont participé à enrichir le manuscrit. Tous les auteurs ont contribué à la rédaction du manuscrit.

\section{REMERCIEMENTS}

Les auteurs remercient le Directeur Général des Eaux et Forêts et toute son équipe pour leurs appui-conseils, ainsi que leur disponibilité. Nous remercions également les autorités communales de la ville de Niamey pour avoir facilité l'accès aux sites de collecte des données.

\section{REFERENCES}

Adamou A. 2005. Parcours migratoire des citadins et problème du logement à Niamey. DEA en géographie, UAM, $157 \mathrm{p}$.

Akobi KI, Amoussou E, Yabi I, Boko M. 2018. Historique de l'évolution anthropique et dynamique du couvert végétal dans la commune de Bantè au Bénin. Int. J. Biol. Chem. Sci., 12(1): 180-194.

DOI: https://dx.doi.org/10.4314/ijbcs.v12i1.14

Alhou B, Micha J-C, Dodo A, Awaiss A. 2009. Etude de la qualité physicochimique et biologique des eaux du fleuve Niger à Niamey. Int. J. Biol. Chem. Sci., 3(2): 240-254.

Harouna M, Machiels P. 1999. Eléments pour un programme urbain, Résultats du survol initial - Programme Urbain de Niamey, déc., 98 p. 
Issiaka Y. 2002. Indicateurs des avantages potentiels d'une zone humide : cas de la plaine d'inondation de N'Dounga-Sebéry (Niger). Mémoire de DEA. Université d'Abobo-Adjamé (RCI), 53 p.

Issiaka Y. 2011. Importances des zones humides du parc National du W du Niger pour les oiseaux d'eau Afro-tropicaux et migrateurs du Paléarctique Occidental. Thèse de Doctorat. Université Abdou Moumouni (Niger), $149 \mathrm{p}$.

Konan EM, Yaokokore-Beibro KH. 2015. Variation temporelle $\mathrm{du}$ peuplement aviaire des écosystèmes lacustres de la ville de Yamoussoukro, centre de la Côte d'Ivoire. Int. J. Biol. Chem. Sci., 9(6): 2566-2581.

DOI:

http://dx.doi.org/10.4314/ijbcs.v9i6.5

Kouagou RS, Apollinaire BM, Mireille ST, Emmanuel S., Armand K. N., Rita O. Jan B. 2018. La biodiversité des parcelles habitées en zone périurbaine à Kinshasa: déterminants socio-biophysiques et représentations. Int. J. Biol. Chem. Sci., 12(3): $\quad 1164-1183 . \quad$ DOI: https://dx.doi.org/10.4314/ijbcs.v12i3.8

Lougbegnon TO, Codjia JC. 2011. Avifaune urbaine de Cotonou et sa distribution en relation avec les facteurs de l'habitat : implications pour l'aménagement écologique de la ville. Afrique SCIENCE, tome 1, vol 07, Cotonou Benin, 116 - 136 pages.

Ndayikengurukiye C. 2005. Inventaire de l'avifaune de la ville de Bujumbura. Relation avec les facteurs de l'habitat. Mémoire de D.E.A en Biologie appliquée. Université de Burundi
OMPO. 2000. Cahier de la formation "Suivi des oiseaux migrateurs et des oiseaux du Niger" Niamey (Niger), 4-13 décembre $2000,163 \mathrm{p}$

Nik B, Ron D. 2015. Guide des oiseaux de l'Afrique de l'Ouest, Delachaux et Niestlé, 511 p.

Sinsin B, Kampman D. 2010. Atlas de la Biodiversité de l'Afrique de l'Ouest, Tome I : Benin. Cotonou et Frankfurt/ main, $726 \mathrm{p}$.

Tini A. 2003. La gestion des déchets solides ménagers à Niamey au Niger : essai pour une stratégie de gestion durable. Thèse en Géographie, Aménagement, Urbanisme. Institut National des Sciences Appliquées de Lyon. 302 p.

Toudjani A. 2012. Contribution à la typologie de la faune aviaire des zones humides de la région de Maradi, Niger: cas des mares de Madarounfa. Mémoire Master2 BGESS, Université de Maradi, 90 p.

Turner WR. 2003. Landscape and Urban Planning 65, p. 149-166.

White JG, Antos MJ, Fitzsimons JA, Palmer GC. 2005. Landscape and Urban Planning, 71, 123-135.

Yaokokoré-Béibro KH. 2001. Avifaune des forêts classées de l'Est de la Cote d'Ivoire. Données sur l'écologie des espèces et effet de la déforestation sur les peuplements. Cas des forêts classées de la Béki et de la Bossématie (Abengourou). Thèse de Doctorat. Université de Cocody-Abidjan (RCI), $245 \mathrm{p}$. 